\title{
Fair Bandwidth Sharing in Network Using FCFS-TP Protocol
}

\author{
Manvendra Singh, Bharat Singh, and Alok Misra
}

\begin{abstract}
In today's scenarios Fair bandwidth Sharing in Distributed system are more complicated and difficult task, every user in network wants to maximize their bandwidth. As we know access to bandwidth is dependent on the Medium Access Control (MAC) protocol, Contention based MAC Protocols are not effective ones and do not results in fairness, also has less utilization of resources. Sharing resource in all users in fair manner is complicated task due to their differences in defining the fairness condition. Their criteria for defining the fairness condition can be based on time, packet, relative weight age etc., but satisfying all criteria is not possible. We have taken some basic criteria for sharing of resources. Hence we proposed FCFS-TP for accessing the medium. In this protocol we have taken time constrained over normal priority. We have analyzed that low priority user who wants to access the medium for less time may get higher priority over high priority user who wants to access the medium for long time.
\end{abstract}

Index Terms - Distributed network, fairbandwidth, medium access control, local area network.

\section{INTRODUCTION}

Traditional protocols for medium access in distributed system assume that each user in the network obey the rules of fair sharing. But due to increasing numbers of users in internet all users want to access more bandwidth by any means. Access to the bandwidth is dependent on the Medium Access Control (MAC) protocol. Access to shared resources is mainly implemented by priority based approaches. A priority is assigned to each task, the task with highest priority is granted exclusive access to the resource. In real-time systems, tasks often face hard deadlines. Due to its predictability in case of collisions, priority-based arbitration mechanisms are commonly used in distributed real-time systems, and applied for scheduling [1] as well as communication in distributed real-time networks [2].

Various MAC Protocols have been proposed for medium access. They can classify into two categories namely Centralized and Distributed.

Centralized MAC Protocol: In centralized network management a host coordinates access to the wired or wireless medium. A node want to transmit must wait until permission to transmit is granted by coordinator node. The mechanism for request for transmission and granting permission for transmission are different in different protocols. Point Coordination Function (PCF) in IEEE
802.11 and A representative of a centralized MAC protocol is the HiperLAN/2 MAC, which is based on polling of terminals (after previous resource requests) are examples of centralized approach.

Distributed MAC Protocol: In distributed network management there is no need of coordinator. In CSMA protocol a node wants to transmit packet only when it does sense the medium idle. In CSMA node independently determines their strategies for transmission of packet. Distributed Coordination Function (DCF) in IEEE 802.11, ALOHA, CSMA/CD and CSMA/CA are examples of Distributed approach.

Above mentioned both approaches have several benefits and have some drawbacks which are given below:

In Centralized approach if a node cannot communicate with Coordinating node then it cannot transmit packets. On the other hand in Distributed approach node can transmit packets even some nodes are missing.

Centralized network management is easy to implement and service differentiation is possible but a single point of failure leads to total network in hazardous position.

Thus we have developed a Semi-Distributed approach for Fair Scheduling. In this approach there is a coordinator which fully centralized in nature and there are several nodes or users those are fully distributed. By developing this type of approach we can manage more fairness in network.

\section{A. Fair Queuing and Other Related Work}

Much researcher has been performed on "fair queuing" algorithms for achieving a fair allocation of bandwidth on a shared link [3], [4]. Consider the system shown in figure 1, where a node maintains several queues which store packets to be transmitted on an output link. A fair queuing algorithm is used to determine which flow to serve next, so as to satisfy a certain fairness criterion. By design, this fair queuing algorithm is centralized, since they are executed on a single node which has access to all information about the flows.

Fair Queuing algorithms approximate the GPS (Generalized Processor sharing) discipline [5]. In GPS discipline, a server serves, say $\mathrm{n}$ flow, which are characterized by their positive weight. Let weight associated with flow $i(i=1, \ldots, n)$ is $\phi_{i}$. Let $W_{i}\left[t_{1}, t_{2}\right]$ be the amount of flow $i$ traffic served in the interval $\left[t_{1}, t_{2}\right]$. Then for the GPS server [5], if flow $i$ is backlogged through $\left[t_{1}, t_{2}\right]$, the flowing condition hold:

$$
\frac{w_{i}\left[t_{1}, t_{2}\right]}{w_{j}\left[t_{1}, t_{2}\right]} \geq \frac{\phi_{i}}{\phi_{j}} \quad \forall j
$$

Manuscript received August 19, 2012; revised October 24, 2012.

The authors are with Department of Computer Science and Engineering, Sri Ramswaroop Memorial College of Engineering and Management affiliated to Gautam Budha Technological University, Lucknow, Uttar Pradesh, India (e-mail: manvendra.mnnit@gmail.com). 
Equality holds if $\mathrm{j}$ is also backlogged in interval $\left[t_{1}, t_{2}\right]$, this implies that the GPS server can "interleave" data from different flow with an arbitrarily fine granularity. The GPS discipline cannot be accurately implemented in practice, since data transmitted in real network is packetized. There are several packet fair queuing algorithm which approximate GPS under the constraint that each packet must be transmitted as a whole [3], [4], [1], [5], [6].these protocol are centralized by nature, as noted above.

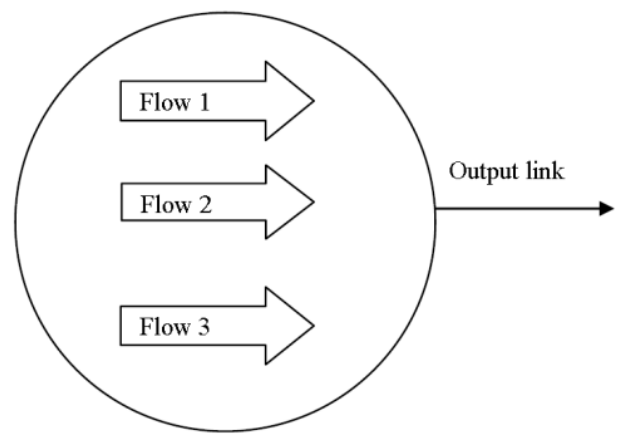

Fig. 1. A node with multiple flow sharing a link

There has been some work on achieving fairness using distributed MAC protocol for wireless network [7],[8]. However, past work on incorporating fairness into distributed protocols has been limited in that these protocol attempt to provide equal fair bandwidth to different nodes. Some game theoretical approach has been proposed for fair medium access in p- persistent MAC strategies, this approach do not provide security against back off attack [9].

Reference [4] Provides security against back off attack but it does not provide bandwidth with respect to the relative weight. There has been work on distributed protocols that take priority, when performing medium access control [9], [10]. However, these protocols do not perform fair allocation of bandwidth. In [11] a work has done on distributed scheduling algorithm for real time traffic on wireless LAN. That work, however, assumes that a flow transmits packet with a constant rate, this type of assumption cannot be made when we are interested to perform fair scheduling.

\section{ASSUMPTIONS}

Our objective is to develop a scheduling in MAC protocol to achieve fairness in wireless LAN (like figure 2). We have following assumption

All nodes in network will be distributed in that all nodes are independent to take their decisions.

There should be a coordinator all nodes must request to the coordinator for priority.

All nodes always maintain their table of priority.

\section{PROPOSED WORKS}

We have proposed a FCFS -Time Priority Based MAC Protocol for fair access of medium in multiple user environments. There are several MAC protocols; those have some drawbacks due to their approaches. Fair Queuing is a centralized approaches that why it has some drawback those are already discussing above. Thus our protocol flows both centralized and distributed approaches that's why we can say this approach semi-distributed approach .All nodes in network wants fair sharing of resources. Sharing resource in all nodes in fair manner is complicated task due to their difference in defining the fairness condition. Their criteria for defining fairness can be based on time, Packet, relative weight etc. But satisfying all criteria is not possible. So we have some basic criteria for fair sharing of resources. Our first Criteria based on FCFS (First Come First Serve) priority and a second criterion is Time based priority. Time is very essential criteria for our proposed protocol. Supposed we have three users in a

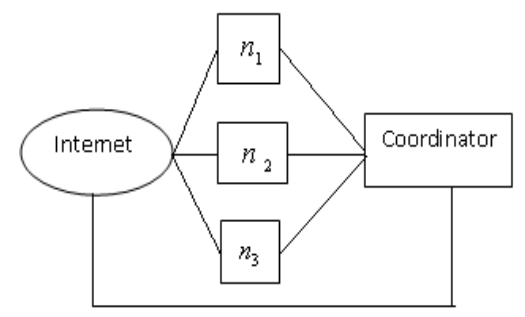

Fig. 2. A simple FCFS-TP based network

Network say $n_{1}, n_{2}$ and $n_{3}$ their priority assign by coordinator $\mathrm{C}$ is $n_{1}=1, n_{2}=2$, and $n_{3}=3$. Now $n_{2}$ and $n_{3}$ want to access the medium for $t_{2}=1 \mathrm{~ms}$ and $t_{3}=2 \mathrm{~ms}$ respectively, but $n_{1}$ wants to use the medium for $t_{1}=6 \mathrm{~ms}$, its means that $n_{1}$ and $n_{2}$ together access medium for $3 \mathrm{~ms}$ while $n_{3}$ alone has taken $6 \mathrm{~ms}$. Its means if we mix the general priority and time based priority $t_{i}$ we can get better fairness result over single criterion based priority.

\section{A. FCFS-Time Priority Protocol Description}

In FCFS - TP protocol, there are $n$ numbers of nodes and a coordinator node $\mathrm{C}$. All nodes wish to send packets to the destination node. Before doing this all node have to achieve a priority. Nodes will send Request to C. Coordinator will assign priority to user (node) on basis of First Come First Serve (FCFS) it means that when a request come to $\mathrm{C}$, it will note down their arrival time, the request which has arrived first, assign with highest priority and send it to the particular node. $\mathrm{C}$ write down the priorities of all node in its priority table. After getting priority, now nodes will choose time slot for using the medium all users or nodes are independent to choose their time slot for transmission. The node which has less time for accessing medium can get higher priority over nodes has large time for accessing medium. Nodes will create new priority after adding priority provided by $\mathrm{C}$ and time for accessing the medium. All nodes broadcast their individual new priority into the network. All receiving nodes create a priority table, nodes will maintain priority of those nodes which has a higher priority and itself. When a node successfully transmits the packet, it will broadcast acknowledgement into the network. After getting ACK all nodes will again maintain the table and remove the priority of node which sends ACK. Nodes will also calculate total time of waiting for their turns. Node set a counter $=$ total waiting time, it will start its countdown timer. When time reaches at 
zero it will wait for ACK of one higher priority node. When it will get the ACK message it will start transmission.

The frame format of the message passing with illustration is given below.

TABLE I: FRAME FORMAT OF FCFS-TP PROTOCOL

\begin{tabular}{|l|l|l|l|l|l|l|}
\hline $\begin{array}{l}\text { Total } \\
\text { time }\end{array}$ & $\begin{array}{l}\text { Request } \\
\text { for } \\
\text { Priority }\end{array}$ & $\begin{array}{l}\text { Assigned } \\
\text { priority }\end{array}$ & $\begin{array}{l}\text { Time } \\
\text { taken by } \\
\text { current } \\
\text { node }\end{array}$ & $\begin{array}{l}\text { Priority of } \\
\text { previous } \\
\text { node }\end{array}$ & $\begin{array}{l}\text { updated } \\
\text { priority or } \\
\text { current } \\
\text { node }\end{array}$ & $\begin{array}{l}\text { A } \\
\text { K }\end{array}$ \\
\hline $\begin{array}{l}20 \\
\mathrm{~ms}\end{array}$ & & $\mathbf{3}$ & $3 \mathrm{~ms}$ & 5 & 6 & 5 \\
\hline
\end{tabular}

In this illustration at first node send request frame to $\mathrm{C}$. Suppose the priority assigned to requested node is 3 , time for transmission chosen by node is $3 \mathrm{~ms}$. We assume that $1 \mathrm{~ms}=$ 1 unit. Now node will calculate new priority or updated priority $=3+3=6$, this updated transmission of all highest priority node. Suppose the total time of transmission is $20 \mathrm{~ms}$, current node (node with priority 6) set its timer for $20 \mathrm{~ms}$ and start countdown when it become zero current node will understand that 5 has releases the medium but node with priority 6 will wait for ACK of node with priority 5.When 6 will get ACK of 5, now medium is free for transmission.

\section{B. Algorithm for FCFS-TP Protocol}

FCFS-TP based on two algorithms first is FCFS for getting general priority from Coordinator and second is time based priority. Those have following steps.

Step 1: At first node $n_{i}$ sends request for getting priority from Coordinating node (C). Where, $i=1, \ldots, n$.

Step 2: After receiving request from the node $n_{i}$ Coordinator will assign there priority on basis of First Come First Serve (FCFS) and send the priority $p_{i}$ to node $n_{i}$.

Step 3: After receiving Priority from $\mathrm{C}$, node $n_{i}$ will choose time $t_{i}$ for accessing the medium.

Step 4: Node $n_{i}$ will calculate new priority

$$
p_{\text {new }(i)}=p_{i}+t_{i}
$$

$n_{i}$ Assumes $1 \mathrm{~ms}=1$ unity.

Step 5: Node $n_{i}$ broadcast $p_{\text {new }}$ into the network.

Step 6: All node in the network Update their new priority and maintain a table of all other nodes priority.

Step 7: Nodes will maintain the tables till

$$
p_{\text {new }(i)}<p_{\text {new }(j)} \forall \mathrm{j}
$$

Step 8: When $p_{\text {new }(j)}=0$; Wait for ACK of $p_{\text {new }(j)}$

Step 9: When ACK receive, transmit packets.

From the above priority based access of medium we see that the performance of fairness depends on FCFS based priority and time for transmission $t$ based priority. That is, the two level of priority input do not independently affect the performance. Their contributions are made through the pair of combined value of $\left(p_{i}, t_{i}\right)$.

\section{Coordinator Policy}

In this section, we discussed about Coordinator policy priority and transmission time will be broadcast into the network. All nodes will also calculate the total time of for real time priority assignment. The policy is as follows:

1) Coordinator will make a table for assigned priority.

2) When a node made their new priority, node sends the time for transmission $t$ and new priority to Coordinator. Coordinator will update the table of that particular node.

3) Coordinator always monitors the nodes behavior and when a node finishes its transmission, Coordinator discards the table of that particular node.

4) Coordinator will not assign priority more than $\mathrm{N}$. Where $\mathrm{N}$ depends on capacity of the network.

Certainly, with this policy, the system fairness performance will improve with FCFS scheduling algorithm.

\section{CONCLUSION}

This Paper considers the issue of fair bandwidth sharing in internet. The objective here is to develop a semi-distributed algorithm for fair packet transmission into the medium. In our approach we provide a solution for sharing bandwidth such that user can get bandwidth on the basis of transmission time and priority. We have proposed a FCFS-TP protocol for accessing the medium, in this protocol we can see that every user has a intention to choose low time for transmission by which they can increase their priority. Users can not deviate from their priority because all users in network have a table of other user's priority and their time slot. Its means that utilization of resource will increase and priority of users in the network is transparent. Our future work is to reduce the massage passing between the nodes and coordinator.

\section{REFERENCES}

[1] P. Goyal, H. M. Vin, and H. Cheng, "Start-time fair queuing: A scheduling algorithm for integrated services packet switching networks," IEEE/ACM Trans. Networking, vol. 5, pp. 690-704, Oct.1997.

[2] G. L. Choudhury and S. S. Rappaport, "Priority access schemes using CSMA-CD," IEEE Transaction on Communication, vol. COM-33, pp. 620-626, July 1985.

[3] J. C. R. Bennet and H. Zhang, "Wf2q: worst- case fair queuing," INFOCOM 96, vol. 2, pp. 120-127, March 1996.

[4] J. Konorski, "A game- theoretic study of CSMA/CA under a backoff attack," IEEE Transaction on Networking, vol. 14, no. 6, pp. 1167-1178, December 2006.

[5] A. K. Parekh and R. G. Gallager, "A generalized processor sharing approach to flow control in integrated services networks: the single-node case," IEEE/ACM Transaction on Networking, vol. 1,pp. 344--357, June 1993.

[6] M. Shreedhar and G. Varghese, " Efficient fair queuing using deficit round robin," in Proc. SIGCOM.95, Cambridge, MA, USA, pp. 231-242, Aug.1995, pp. 231-242.

[7] V. Bharghavan, A. Demers, S. Shenker, and L. Zang, "MACAW: A media access protocol for wireless LANs," in Proc. ACM SIGCOMM, , August 1994, p. 212-225

[8] M. Gerla, K. Tang, and R. Bagrodia, "TCP performance in wireless multihop networks," in Proc. IEEE Workshop Mobile Computing Systems and Applications (WMCSA), Feb. 1999, pp. 41-50.

[9] S. Rakshit and R. K. Guha, "Fair bandwidth sharing in distributed systems: a game-theoretic Approach," IEEE Transaction on Computers, vol. 54, no. 11, pp. 1384-1393, November 2005. 
[10] S. M. Sharrock and D. H. Du, "Efficient CSMA/CD-based protocols for multiple priority classes," IEEE Transaction on Computers, vol. 38, pp. 943-954, July 1989.

[11] J. L. Sobrinho and A. S. Krishnakumar, "Real-time traffic over the IEEE 802.11 medium access control layer," in Proc. Bell Labs Technical Journal, Autumn 1996, pp. 172-187.

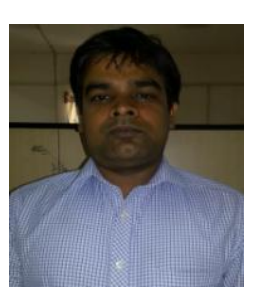

Manvendra Singh obtained his B.Tech in 2007 in Information Technology, Department of Computer Science and Information Technology from West Bengal University of Technology, Kolkata, West Bengal, India and M.Tech in Information Security in 2010, Department of Information Technology form National Institute of Technology, Durgapur, west Bengal, India. $\mathrm{He}$ is currently Assistant Professor in Computer Science and Engineering department in Sri Ramswaroop Memorial College Of Engineering and Management affiliated to Gautam Budha Technological University, Lucknow, Uttar Pradesh. His current research area is Mobile Computing, Distributed systems and Computer Network.

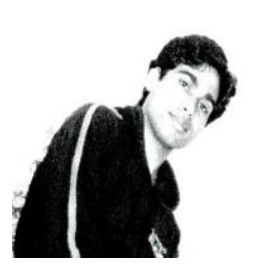

Bharat Singh obtained his B.Tech in 2008 in Computer Science and Engineering , Department of Computer Science and Engineering in, from Uttar Paradesh Technical University and M.Tech in Information Technology in 2010, Department of Computer Science and Engineering form National Institute of Technology, Durgapur, west Bengal, India. He was Assistant Professor in Computer Science and Engineering department in Sri Ramswaroop Memorial College Of Engineering and Management affiliated to Gautam Budha Technological University, Lucknow, Uttar Pradesh. He is currently pursuing $\mathrm{PhD}$ from Indian Institute of Information Technology Allahabad, India. His current research area is Data mining, Wireless sensor.

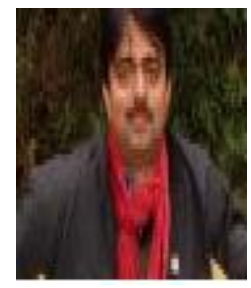

Alok Misra obtained his M.Tech in Computer Science and Engineering, Department of Computer Science and Engineering from Indian Institute of Technology, Guwahati, Assam, India .He is currently Assistant Professor in Computer Science and Engineering department in Sri Ramswaroop Memorial College Of Engineering and Management affiliated to Gautam Budha Technological University, Lucknow, Uttar Pradesh. His current research area is Mobile Computing and Computer Networks. 